\title{
Calculation the options of cracks in the earth dam
}

\author{
Yulia Ibraeva $^{1, a}$, Georgiy Bulatov ${ }^{2}$ and Philipp Tarasevskii ${ }^{3}$ \\ ${ }^{1}$ Peter the Great St.Petersburg Polytechnic University, 195251,St. Petersburg, Russian Federation
}

\begin{abstract}
The article presents the calculation of the limit depth of cracks in the dam, the calculation of step cracks in the dam, the calculation of full disclosure, the calculation of the direction of the cracks in the dam, the position of the first crack and their natural clogging. As well in this article has been shown all the necessary formulas for these calculations.
\end{abstract}

\section{Introduction}

Despite the considerable progress made in the soil construction of dams, reserves to reduce the cost of construction of earth dams and increase their strength filtration completely exhausted. Mainly, in our opinion, is to increase reserves crack resistance ground shields, cores, and ensuring the principle of equal strength of all elements of the dam by further improving the design and technological solutions.

\section{Calculation of limiting the depth of cracks in the dam}

The limiting depth the propagation of vertical crack [1-3]

$$
h_{c . \lim }=\frac{2 \cdot\left(C+C_{w}\right)}{\gamma_{g r} \cdot \operatorname{tg} \beta_{a}}
$$

Where: $\mathrm{C}$ - clutch of soil in shear, $\mathrm{C}_{\mathrm{w}}-$ additional clutch which equivalent to support actions of filtration forces and called overpressure of water in the cavity of cracks (at the phenomenon of hydraulic fracturing), $\gamma_{\mathrm{gr}}-$ unit weight the soil of dam, averaged over the height of the crack, $\beta_{\mathrm{a}}-$ the angle of the active soil collapse.

$$
\beta_{a}=\frac{\pi}{4}-\frac{\varphi}{2}
$$

Where: $\varphi$ - the angle of internal friction of soil.

$$
C_{w}=\eta_{c} \cdot p_{w}
$$

Where: $\eta_{\mathrm{c}}-$ average value of water pressure in the cavity cracks, $\mathrm{p}_{\mathrm{w}}-$ the coefficient of additional clutch from water pressure (in the first approximation, equal to 0.1 ).

\footnotetext{
${ }^{\text {a} C o r r e s p o n d i n g ~ a u t h o r: ~ u l c h i 412 @ m a i l . r u ~}$
} 
By separating at the crestal area of the dam on the surface and underwater, and taking the water pressure in the cavity of the crack below the water averaged hydrostatic, we write

$$
h_{c . l i m}=\frac{2 C-h_{f}\left[\left(\gamma_{f}-\gamma_{\text {sus }}\right) \cdot \operatorname{tg} \beta_{a}+\eta_{c} \cdot \gamma_{w}\right]}{\gamma_{\text {sus }} \cdot \operatorname{tg} \beta_{a}-\eta_{c} \cdot \gamma_{w}}
$$

Where: $h_{f}-$ freeboard of the dam, $\gamma_{f}$ и $\gamma_{\text {sus }}-$ densities and soil respectively on the surface and in suspension in the water condition, $\gamma_{\mathrm{w}}$-specific gravity of water.

Eq. 1 for $\mathrm{C}_{\mathrm{w}}=0$ is recommended by many authors to determine the depth of cracks as well as the height of the maximum sustainable vertical ground slope [1-17].

It can also be obtained from the condition of soil strength in compression at the base of the vertical slope in the form of

$$
h_{v e r t}=\frac{R_{c}}{\gamma_{g r}}
$$

Where: $R_{c}-$ strength of the soil compression by the limit equilibrium theory [11-19].

The height of the vertical slope is defined as

$$
\mathrm{h}_{\mathrm{vert}}=\frac{3.8 \cdot \mathrm{C}}{\gamma_{\mathrm{gr}}}
$$

Were considered above options for the conditions in the plane problem. But when the transverse cracks in the dam equilibrium problem of vertical slope is voluminous, and for its, taking into account increased risk of fractures, we write

$$
\mathrm{h}_{\text {c.lim }}^{\prime}=\frac{2 \cdot\left(\mathrm{C}+\mathrm{C}_{\mathrm{w}}\right)}{\gamma_{\mathrm{gr}} \cdot \operatorname{tg} \beta_{\mathrm{a}}} \cdot \mathrm{K}_{\mathrm{v}}
$$

Where: $\mathrm{K}_{\mathrm{v}}-$ coefficient taking into account the spatial tasks.

Assuming that there exist flat surfaces collapse obtain

$$
K_{v} \cong \frac{l}{l_{0}}=\frac{B+m \cdot h_{c . l i m}}{B+2 \cdot m \cdot \frac{h_{c . l i m}}{3}} \cdot \frac{\sin \beta_{a}}{\sin \beta_{a 0}}
$$

Where: 1 и $\mathrm{l}_{0}$ - calculated lengths of the surface of collapse equal to the ratio of body volume of collapse toward to area surface collapse, respectively for flat and volume tasks, B and $m$-width of the crest of the dam and the average inception of its slope in the direction of the axis of the crack, $\beta_{\mathrm{a} 0}-$ the angle of active caving ground in the volumetric conditions task (may be taken as approximately $\beta_{\mathrm{a}}$ ).

\section{Calculation step cracks in the dam}

Average cracking step in analogy with the width of prism the active collapsing soil is determined based on the data [20] by one of the following expressions

$$
\begin{gathered}
a_{c}=h_{c . l i m} \cdot \eta_{a} \cdot \xi_{r g}, \\
\psi_{m} \cdot h_{c . l i m} \text { at } h_{l}<h_{c . l i m}
\end{gathered}
$$

Where: $h_{\text {c.lim }}$ - maximum depth of crack propagation, $\eta_{a}-$ coefficient which takes into account the widely observed random factor (scatter) in the formation of cracks, expressed in a significant natural irregularities of the distance between them (approximately $1 \ldots 2$ ), $\xi_{\text {rg }}$ - function unloading solid ground in the event of a crack in it, taken as a 


$$
\xi_{r g}=\operatorname{tg} \beta_{a}
$$

Where: $\beta_{\mathrm{a}}$ - active caving angle of soil, $\psi_{\mathrm{m}}$ - coefficient which reflecting layer monolithic taken equal to $1,2 \ldots$ at the transition from unconsolidated soil to little connected dense soils, given that individual rocks to approximate an average of the cubic form, $\mathrm{h}_{\mathrm{l}}-$ thickness of the layer of soil (for example dams or soil impervious element) [21-24].

The place of potential cracks will be any weakened vertical section in the dam or in its base, defined throughout their hydrogeological history at the material time: cracks in the layer, ground interlayer, thinning, chink, and conjugation of elements structures, technology seams and others.

Note. At the junction of dirt and concrete parts of the building or in the sectional seams of deformation extension of the earth surface will be compensated (absorbed) by the appropriate disclosure of cracks, which calculated similarly fissures.

Often in the base of dams under a layer unconsolidated sediments overlain layer of solid rock. In this case it is useful to check the parameters of cracks on the stratum, as it can make a decisive contribution to the character of crack in the dam body. In one of the options, this calculation should be equal to the pitch of cracks at the base formation of the hard rock. In the presence in the base laminate thickness desired verification options cracking and of other layers.

A large range of properties of natural soils and including their strength characteristics, can also introduce the concept of the minimum step of cracks.

For example, the minimum step of cracks on the conditions of fracture cracks dirt console

$$
a_{t . k .}=h_{c} \cdot \sqrt{\frac{R_{p}}{3 \cdot \gamma_{g r} \cdot h_{c}}}
$$

Or conditions shear of console

$$
a_{t . k .}=\frac{C}{\gamma_{g r}}
$$

Where: $h_{c}-$ crack depth, $\mathrm{R}_{\mathrm{p}}$ и $\mathrm{C}$-strength of the soil, respectively, and the tensile shear, $\gamma_{\mathrm{gr}}-$ unit weight of soil.

Natural to assume that the extent value of deformations of the step cracks will be changes accordingly. Approximate regularity of marked changes proposed below. By analogy with the expression permanent deformation in the form of CES

$$
R^{*} \cdot \varepsilon^{*}=C_{R}^{*}
$$

We can write the expression for constant cracking

$$
a_{c}^{*}-\varepsilon^{*}=C_{a}^{*}
$$

From a comparison of these relations we obtain

$$
a_{c}^{*}=R^{*} \cdot C_{c}^{*} \geq a_{c \min }
$$

At a constant pitch of cracks

$$
C_{c}^{*}=\frac{C_{a}^{*}}{C_{R}^{*}}
$$

Where: $a_{T \min }-$ minimum step of cracks.

\section{Calculation of the total crack opening}

In accordance with the entire disclosure of cracks 


$$
\delta=\delta_{0}+\Delta \delta
$$

If

$$
\delta_{0}=\delta_{p}+\delta_{\text {man }}+\delta_{\text {oth }}
$$

Where: $\delta_{0}$ - crack opening before erosion, $\Delta \delta$-the same additional, in the process of erosion, $\delta_{\mathrm{p}}, \delta_{\text {man }}, \delta_{\text {oth }}-$ the same against part-time, managers and other factors [24-27].

Crack opening of part-time work

$$
\delta_{p}=\left(\varepsilon^{*}+\frac{\Delta R}{R^{*}}+\varepsilon_{m a n}-\varepsilon_{k}\right) \cdot a_{c},
$$

If

$$
\varepsilon_{\text {man }}=\frac{R_{p}}{E_{p z . p}} \cdot \lambda(z)
$$

Where: $\varepsilon^{*}$-the expected horizontal deformation of elongation, $\varepsilon_{\text {man }^{-}}$deformation of elastic recovery during unloading from stretching, $\mathrm{E}_{\text {рг. }}{ }^{-}$the modulus of deformation of soil which is corresponding to it, $\lambda(\mathrm{z})$ - the function of restore the elastic deformations.

In a first approximation

$$
\lambda(z)=\frac{1}{3} \cdot\left(1-\frac{z}{h_{c}}\right) \cdot\left(1-\mu^{2}\right) \geq 0
$$

Where: $\mathrm{h}_{\mathrm{c}}-$ the intermediate depth of the crack propagation.

Entering values into Eq. 20 and equating the left side to zero, and of assuming that $\mathrm{z}=\mathrm{h}_{\mathrm{c}}$, taking into account other influences obtain

$$
h_{T}=\frac{\varepsilon^{*}+\frac{\Delta h}{R^{*}-\varepsilon_{l}\left(1-\mu^{2}\right)+\varepsilon_{o t h}+\varepsilon_{\text {man }}}}{\frac{1}{R^{*}}+\frac{\xi \cdot \gamma_{g r} \cdot K_{a}}{E_{p z . c}(T)}\left(1-\mu^{2}\right)} \leq h_{c . l i m}
$$

With the notation introduced earlier. In practice, part-time work has been cases where a single point at the beginning of CES undergoes compressive strain $\varepsilon_{1}^{*}$, and then lengthening $\varepsilon_{2}^{*}$. In this case it is recommended to take

$$
\varepsilon^{*}=\left[\varepsilon_{2}^{*}-\varepsilon_{l}^{*}\left(1-\frac{E_{c}}{E_{p z . c}}\right)\right]
$$

Where: $E_{c}, E_{\text {рг.c }}-$ the modulus of soil deformation respectively in compression and unloading of the compressive stress which are caused through compressive strain.

\section{The calculation of the direction of cracks in the dam}

Dams can change the direction of cracks developing on the Earth's surface (CES). Deflection angle $\chi$ line breaks in the dam plan (Fig. 1) from the normal to the major principal elongation $\varepsilon^{*}$ (i.e. in terms of the crack deviation) is determined from the condition

$$
\frac{\varepsilon}{F}=\max
$$


Where: $\varepsilon$ - component strain elongation in the direction normal to the line break, $\mathrm{F}$ - crosssectional area of the dam along the line break

$$
\begin{gathered}
\varepsilon=\varepsilon^{*} \cdot \cos ^{2} \chi, \\
F=\frac{H_{d}}{2}\left(B_{g r}+\frac{B_{g r}+H_{d}\left(m_{B}+m_{H}\right)}{\cos (\theta-\chi)}\right)
\end{gathered}
$$

Where: $\varepsilon^{*}$ - most importantly elongation, $\chi$ - deviation in terms of a crack, $\mathrm{H}_{\mathrm{d}}$-height of the dam,

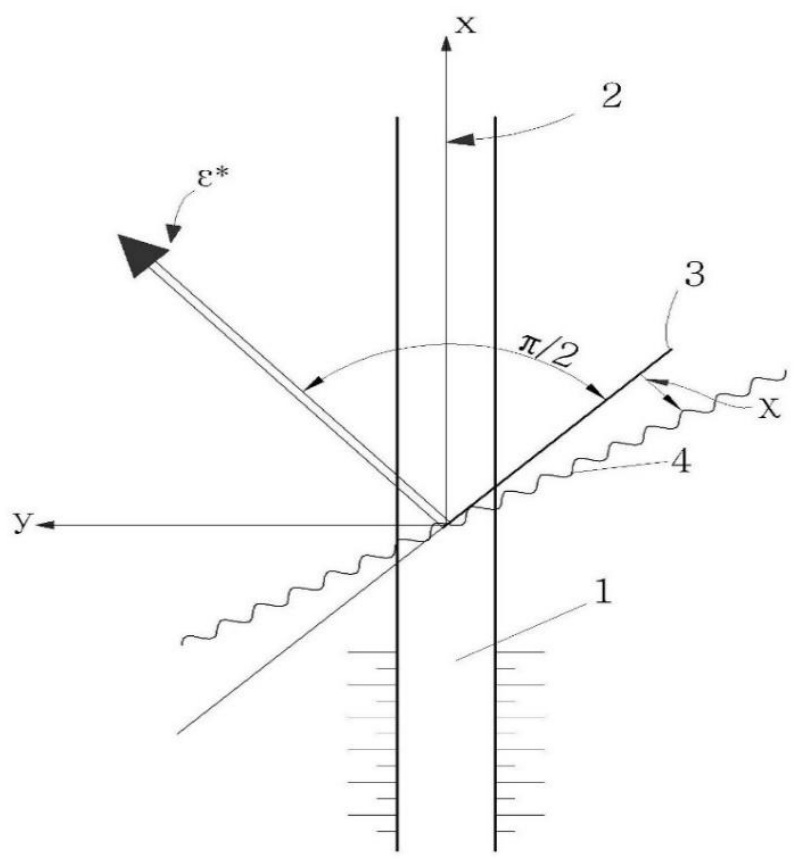

Figure 1. The scheme of deformation of the dam in terms of: 1 - crest of the dam; 2 - axis of the dam; 3 normal to the main elongation; 4 - axle cracks

$\mathrm{B}_{\mathrm{gr}}-$ width of the dam, $\mathrm{m}_{\mathrm{B}}$ и $\mathrm{m}_{\mathrm{H}^{-}}$foundations, the upper and lower slope, $\theta$ - the angle of deflection of the main extension at longitudinal axis of the dam [18-25].

Of the conditions for the crack deviation we obtained the equation

$$
\frac{B_{g r}}{B_{g r}+H_{d}\left(m_{B}+m_{H}\right)} \cdot \cos (\theta-\chi)+1-\frac{\operatorname{tg}(\theta-\chi)}{2 \operatorname{tg} \chi}=0
$$

The first term may be taken as approximately 0 . From equation that

$$
\chi \cong \frac{\theta}{3}
$$

In the vertical plane, we can assume that in the side prisms of dam the cracks will be deviate from the vertical on the direction by an angle of slope (deviation in a vertical sectional view of a crack) [24-26].

$$
\psi \leq \beta_{a}
$$




\section{Status of the first cracks}

Location and direction of the crack is determined by well-known law of nature "where the thin, there and tear." Initially, there is only one (the first) crack - and this is also the law. And the feature of it is that the appearance of subsequent fractures is essentially determined by the first of them.

The first crack may not coincide with the position of the maximum elongation CES, and deviate in the direction of various kinds weakened regions due to multiple causes of natural and man-made, non-ideal creations of both nature and human hands.

For example, the place of appearance the cracks on the slope of the dam may be incoming (internal) angle berm, etc. Both obvious and subtle at first glance factors can significantly alter the picture of fracture. Sometimes arise and unexpected phenomena, such as the complete cracking dam with the rapid emptying of the repository. Of course, as elsewhere, in this case also can help only a closer analysis of the system.

\section{The natural cracks clogged}

Rainwater flows periodically come to life on the crest of dam mainly in the ruts of transport, at a meeting with cracks fell through in them and spread out over the slopes. These flows are accompanied by phenomena of transport (erosion-per wash) soil particles, which are the analogous phenomena of clogging of cracks in the pavement slabs or parquet floor cracks.

The phenomenon of clogging reminiscent the central alluvium with the axis predominantly on the middle ridge width. The density of the material filling of the cavity thin cracks will be small due to the appearance the effects of silage and loose appearance at elevated heterogeneity filling.

At the beginning washed away the small particles that fill up the tip of crack. Then wash up in the cavity of the new layers of soil, washed from the crest of the dam. In heavy rain on the slopes are possible the formation of micro ravines eroded cracks in the upper part (the rivers is usually confined to the geological fractures).

In spring can be partially flood of cracks due to water-logged soil. At the end of the dry season, the crack may become more pronounced. Reliability natural clogging cracks will be determined by the ratio of the rate of formation of the tampon to the speed of progress the cavity of crack. Generalized criterion of adequacy clogging represented as

$$
\Pi_{i}(T) \geq\left[\Pi_{i}(T)\right]
$$

Where: $\Pi_{\mathrm{i}}(\mathrm{T})$ - expected parameters tamponing cracks (in brackets and their allowed values of the terms of reliability), $i=1$ - thickness of the tampon, $i=2$ - height of the tampon, $i=3-$ length, $i=4-$ permeability and density, $i=5-$ its strength of filtration.

Expected parameters clogging cracks are determined based on the theories of climate predictions water erosion and wash. The valid options are evaluated according to the criteria of reliability, below.

\section{Results and Discussion}

The result of the article is the formula by which, in the earth dam are determined: the depth of the cracks, the value of disclosure, presumably the direction of opening and a step appearance. Using this methodology can be compute required constructively-technologic protection measures.

\section{Conclusion}

Thus, we can calculate the parameters of cracks appearing in the earth dam and to predict the appearance of the first crack. Due to the calculation of crack size and step their formation we can introduce the event name for the specified concrete fracture parameters, and to avoid their occurrence in groundwater dam and as a consequence of further negative impact on the body of a subsurface dam. 


\section{References}

1. R. Usmanov, M. Rakočević, V. Murgul, N. Vatin, Applied Mechanics and Materials, 633-634, 927-931 (2014)

2. R. Usmanov, I. Mrdak, N. Vatin, V. Murgul, Applied Mechanics and Materials, 633-634, 932935 (2014)

3. M. Jocovic, B. Melovic, N. Vatin, V. Murgul, Applied Mechanics and Materials, 678, 644-647 (2014)

4. Y. Sonoda, Applied Mechanics and Materials, 566, 10-25 (2014)

5. E. Panulinová, S. Harabinová, Advanced Materials Research, 969, 245-248 (2014)

6. S. Harabinová, E. Panulinová, Advanced Materials Research, 969, 208-211 (2014)

7. M. Sharp, Y. Seda-Sanabria, E. Matheu, Applied Mechanics and Materials, 82, 428-433 (2011)

8. I. Mrdak, M. Rakočević, L. Žugić, R. Usmanov, V. Murgul, N. Vatin, Applied Mechanics and Materials, 633 - 634, 1069-1076 (2014)

9. D. Kubečková, Advanced Materials Research, 1020, 883-887 (2014)

10. G. Bulatov, V. Teleshev, V. Leonov, International Water Power \& Dam Construction, 117, 111115 (2003)

11. D. Hilyard, International Water Power \& Dam Construction, 5, 20-22 (2010)

12. S. Bortkevich, Power Technology and Engineering, 5, 287-292 (2009)

13. M. Jocovic, B. Melovic, N. Vatin, V. Murgul, Applied Mechanics and Materials, 678, 644-647 (2014)

14. N. Vatin, N. Lavrov, N. Korzhavin, Applied Mechanics and Materials, 641-642, 353-358 (2014)

15. V. Vladimirov, Yu. Zaretskii, V. Orekhov, Power Technology and Engineering, 3, 161-166 (2003)

16. S. Shkol'nikov, I. Sekisova, Power Technology and Engineering, 5, 331-339 (2008)

17. V. Bukhartsev, M. Petrichenko, Power Technology and Engineering, 46 (3), 185-189 (2012)

18. G. Bulatov, Trudy LPI, 375, 91-94 (1981)

19. V. Istomina, V. Burenkova, G. Mishurova, The filter strength of clay soils (Stroyizdat, 220, 1975)

20. A. Bougrov, V. Markevich, Gidrotekhnicheskoye stroitel'stvo, 4, 24-26 (1980)

21. G. Bulatov, N. Vatin, D. Nemova, Y. Ibraeva, P. Tarasevskii, Applied Mechanics and Materials, 725-726, 342-349 (2014)

22. V. Deković, A. Andelković, N. Milošević, G. Gajić, M. Janić, Carpathian Journal of Earth and Environmental Sciences, 8 (2), 107-112 (2013)

23. L. Rasskazov, M. Smirnova, Power Technology and Engineering, 48 (2), 85-88 (2014)

24. A. Bauer, S. Haug, WasserWirtschaft, 104 (4), 28-33 (2014)

25. J. Guo, I. Hasan, P. Graeber, Springer Series in Geomechanics and Geoengineering, 2015, 195209 (2014)

26. M. Balzannikov, M. Rodionov, International Journal on Hydropower and Dams, 20 (6), 60-63 (2013)

27. A. Feringer, N. Kabanov, Power Technology and Engineering, 46 (5), 380-383 (2013) 